\title{
Research on Dynamic Coupled Characteristics of A Tracked Vehicle Gearbox
}

\author{
Hui Liu ${ }^{1,2}$ * \\ 1 School of Mechanical Engineering, Beijing Institute of Technology, Beijing 100081, P. R.China \\ 2 Science and Technology on Vehicle Transmission Laboratory, Beijing 100081, P. R. China \\ Changle Xiang ${ }^{1,2}$ \\ 1 School of Mechanical Engineering, Beijing Institute of Technology, Beijing 100081, P. R.China \\ 2 Science and Technology on Vehicle Transmission Laboratory, Beijing 100081, P. R. China \\ Shengping Fu \\ School of Mechanical Engineering, Beijing Institute of Technology, Beijing 100081, P. R.China
}

Received 20 August 2011

Accepted 25 November 2011

\begin{abstract}
A tracked vehicle gearbox is divided into two subsystems-housing and gear train. Dynamic behaviors of the two subsystems are coupled practically. And the coupled characteristics describe the integrative dynamic behaviors of gearbox. This study proposes a coupled simulation model to investigate the interrelationship between dynamics of two subsystems. Multi-source excitations are numerically calculated to provide boundary conditions. The flexibility of transmission shafts and housing is mathematically described based on mode superposition. The coupled dynamic characteristics are analyzed with dynamics simulation computation. The flexibility of housing is one of the main causes to induce the fluctuation of dynamic responses of transmission shafts. The experimental results show that the proposed method is accurate through comparison of simulation results and test data.
\end{abstract}

Keywords: Dynamic Coupled Characteristics, Gearbox, Tracked Vehicle, Multi-source Excitations, Multi-body Dynamics

\section{Introduction}

With the number of cars dramatically increasing, improve the traffic safety and reduce traffic accident becomes the focus of the research1. 2The traffic safety has the close relationship with the performance of the vehicular transmission. The dynamic characteristics simulation of transmission are effective ways to optimize the transmission performance and ensure the reliability.
The vehicle gearbox is a complicated flexible mechanical system, which consists of the transmission system in consideration of transmission shafts, gear sets, supporting bearings etc. And the structural system mainly includes housing, which supports the transmission system. So housing flexible deformation is the input disturbance variable for the transmission system, resulting in the variation of system dynamics behaviors. The housing responds to the movement of transmission system. Therefore, on the dynamics study of gearbox, it is not only needed to consider the

*Corresponding author: lh@bit.edu.cn 
dynamic characteristics of transmission system, but also to analyze the interaction of structural system and transmission system. Besides aforementioned factors, external and interior excitations also lead to the system instability. Consequently the whole system must be regarded as one object to construct the analysis model of gearbox.

The gearbox dynamics has been extensively studied. Y.Wang and W.J.Zhang ${ }^{3}$ developed a stochastic vibration simulation model of transmission systems with consideration of speed-dependent random errors. The vibration of gear transmission systems was analyzed with consideration of the influence of the time-variant stiffness, loads, and gear transmission errors. Grzegorz Litak and Michael I.Friswell ${ }^{4}$ examined the dynamics of gear sets with various faults applying standard methods for nonlinear systems with various faults in meshing stiffness. Tahar Fakhfakh, Fakher Chaari and Mohamed Haddar $^{5}$ constructed a one-stage spur gear transmission including rigid body and elastic body. It was presented that spectrum and cepstrum analysis techniques were very efficient for localized and distributed defects. Chang-Jian and Chen ${ }^{6,7,8}$ studied flexible rotor supported by journal bearings under non-linear suspension, and discovered non-periodic responses of rotor-bearing system to help people escape undesired motions. Saeed Ebrahimi, Peter Eberhard ${ }^{9}$ incorporated gear wheels into multi-body systems and simulated contact between them. Because of the teeth and the gear wheel body being modeled as being rigid and the connection between each other as the elastic elements, an efficient algorithm was presented to find the effects of multi-tooth contact. Chan IL Park ${ }^{10}$ studied a simplified model describing gear, shaft, bearing and housing to predict analytically the vibration from the helical gear due to an axial excitation of helical gears. Numerical simulation of the overall dynamic behavior of a parallel helical gear transmission was studied by Mohamed Slim Abbes, Tahar Frakhfrakh etc. Reference $11,12,13$ and 14 discussed the dynamic behavior of gearbox housing. Therefore, the current dynamics researches of gearbox mainly focused on the independent analyses of gear train system and dynamics behaviors of gearbox housing, neglecting coupled dynamic characteristic.

Chongqing University ${ }^{15,16}$ established the finite element model about gearbox. Natural mode and dynamic response of housing surface were deeply studied, but it did not discuss the affection of housing flexibility on dynamics behaviors of transmission.

Consequently it is significant to constitute dynamics analysis model of gearbox containing flexible gearbox housing and transmission shafts. This work was concerned with the numerically simulation of overall dynamic coupled behaviors of gearbox with the consideration of multi-source excitations.

\section{Description of the Studied Gearbox}

A tri-freedom fixed axis multistage gearbox in a certain tracked vehicle is investigated. Fig.1. shows the gearbox model. The engine power is input from bevel gear as Fig.1 shows. Gear sets are main components for power transmission. Bearings are assembled on the box to support transmission shafts. So the gear meshing force passes from shafts to bearings and reflected by the dynamic response of box finally. The shift exchange is realized by operating the wet clutch. The introduction of shifting logic with corresponding operating elements is shown in Table 1. "O”represents the lock of clutch.

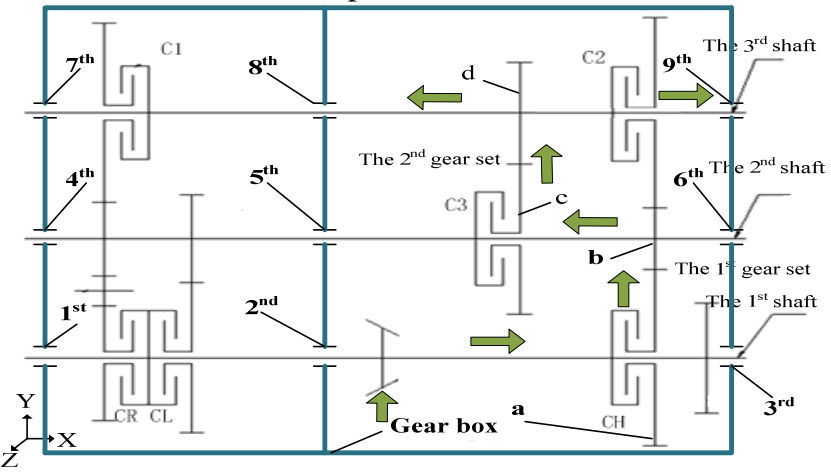

Fig.1. Gearbox model

Table 1. Shifting logic of the gearbox

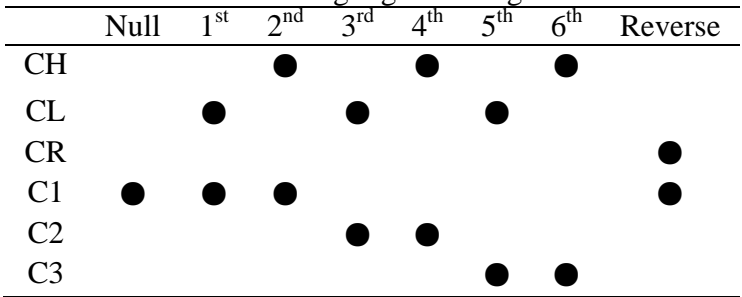

This work was concentrated on the study of dynamic characteristics of gearbox under some fixed shifting, not considering the transition situation. Owing to the unstable property of system under high speed 
condition, the $6^{\text {th }}$ shifting was selected to analyze. And the power flows as arrowheads pointing in Fig.1.

For this system, the housing flexibility coupling with the dynamics function of gear transmission system is realized under multi-source excitations based on finite element theory and multibody dynamics. The analysis model and simulation results were validated by test.

\section{Modeling Theory Foundation}

Dynamic sub-structure method is very efficient to analyze the complicated mechanical system and structural system ${ }^{17}$. Based on the resolution principle of dynamic sub-structure method, gearbox is decomposed into four independent substructures-transmission shafts, gear sets, supporting bearings and housing.

It is crucial to establish displacement constraints and boundary conditions of each substructure before analysis. For transmission shafts, interface nodes are placed at the geometry center of transmission gear sets, clutches and supporting bearings. Its generalized displacement vector $\{\delta\}_{s}$ is:

$$
\{\delta\}_{s}=\left\{\left\{\delta_{j}\right\}_{s}^{T}\left\{\delta_{n j}\right\}_{s}^{T}\right\}^{T}
$$

where $\left\{\delta_{j}\right\}_{s}$ is the interface nodes displacement vector, $\left\{\delta_{n j}\right\}_{s}$ is the non-interface nodes displacement vector.

For gear pairs, interface nodes are placed at the geometric center of gear body. Its generalized displacement vector $\{\delta\}_{c}$ is:

$$
\{\delta\}_{c}=\left\{\left\{\delta_{z}\right\}_{c}^{T}\left\{\delta_{c}\right\}_{c}^{T}\right\}^{T}
$$

where $\left\{\delta_{z}\right\}_{c}$ is the interface nodes displacement vector of driving gears, $\left\{\delta_{c}\right\}_{c}$ is the interface nodes displacement vector of driven gears.

For supporting bearings, interface nodes were placed at the connection interface center of bearing with shaft and housing. Its generalized displacement vector $\{\delta\}_{z}$ is:

$$
\{\delta\}_{z}=\left\{\left\{\delta_{s}\right\}_{z}^{T}\left\{\delta_{x}\right\}_{z}^{T}\right\}^{T}
$$

where $\left\{\delta_{s}\right\}_{z}$ is the displacement vector of nodes connecting with bearing and shaft, $\left\{\delta_{x}\right\}_{z}$ is the displacement vector of nodes connecting with bearing and housing.

For housing, multi nodes are adopted to combine with the interface where bearings are assembled. Its generalized displacement vector $\{\delta\}_{x}$ is:

$$
\{\delta\}_{x}=\left\{\left\{\delta_{j}\right\}_{x}^{T}\left\{\delta_{n j}\right\}_{x}^{T}\right\}^{T}
$$

where $\left\{\delta_{j}\right\}_{x}$ is the interface nodes displacement vector, $\left\{\delta_{n j}\right\}_{x}$ is the non-interface nodes displacement vector.

On the assumption that there is no elastic deformation at connection interface of substructures, displacement constraint condition exists between correlative adjacent interfaces, and mathematical expression is:

$$
\left\{\begin{array}{l}
\left\{\delta_{z a}\right\}_{c}=\left\{\delta_{z a}\right\}_{s} \\
\left\{\delta_{z b}\right\}_{c}=\left\{\delta_{z b}\right\}_{s} \\
\left\{\delta_{j}\right\}_{a}=\left\{\delta_{a j}\right\}_{z} \\
\left\{\delta_{x}\right\}_{z}=\left\{\delta_{n j}\right\}_{x}
\end{array}\right.
$$

where subscript a represents driving gear, subscript b represents driven gear, subscript $\mathrm{j}$ represents bearing number.

Based on dynamics equations of every substructure and displacement constraint conditions between interfaces, the whole system analysis model is constructed and solved.

\section{Finite Element Analysis of Gearbox Housing}

The housing is the supporting component of gearbox. The whole housing is made up of top housing and bottom housing, left cover and right cover. Its shape is abnormal and asymmetric.

\subsection{Finite element model and mode analysis}

The studied housing is a thin-wall complicated special component, containing many geometrical elements such as stiffener, boss, chamfer, bolt connection hole and so on. After simplifying and amending the geometrical model, finite element model of the housing (shown as Fig.2) is established via element selection, the description of components connection and the definition of material properties and boundary condition.

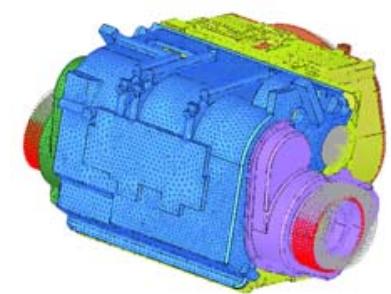

Fig.2. Finite element model of gearbox housing 
To consider the housing flexibility, namely obtaining natural frequency and modal shape of housing, free mode of gearbox housing is analyzed. Fig. 3 shows the fifth order and the seventh order mode of housing.

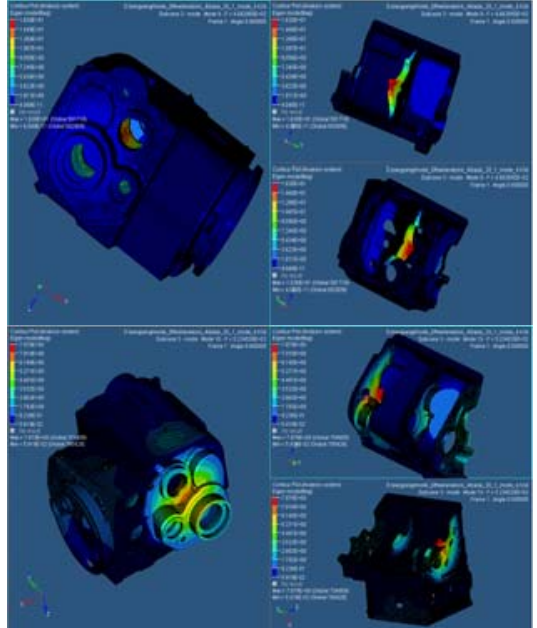

Fig.3. The $5^{\text {th }}$ and $7^{\text {th }}$ order mode of housing

\subsection{Experimental verification}

Because of the advanced development of hamming method, it was used to research on the modal test of the housing.

8206 type excitation hammer of Danish B\&K Company, 4326 type three-direction accelerometer, SC10DC-UTP type data collection equipment of LMS company and LMS Test. Lab (data analysis software) were selected in this experiment. The modal parameters characterizing gearbox dynamics could be gained. The principle of selecting test points was to avoid the vibration node and reflect the vibration mode well. According to this principle, measuring points were arranged as Fig.4 shows.

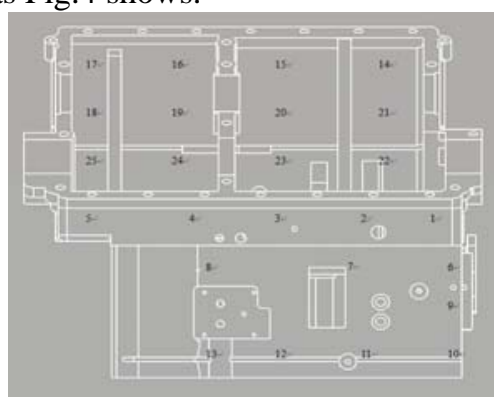

Fig.4. The arrangment of test points in down housing

To avoid the effect of environmental vibration and supporting stiffness on the test results, free hanging method was adopted. In that way, the measured component was in a free state. Out of taking rigid modes into consideration, comparison of the modal parameters resulted from experiment and numerically simulation was executed. Table 2 lists the comparison results.

Table 2. Comparison of housing natural frequency between

\begin{tabular}{cccc}
\multicolumn{4}{c}{ the experimental results and simulation results } \\
\hline Order & Test/Hz & Simulation/Hz & Error/\% \\
\hline 1 & 367.2 & 376.8 & 2.57 \\
2 & 391.4 & 385.1 & 1.64 \\
3 & 419.1 & 424.0 & 1.16 \\
4 & 434.5 & 452.1 & 3.88 \\
5 & 458.6 & 468.3 & 2.07 \\
6 & 498.9 & 487.8 & 2.28 \\
7 & 531.1 & 523.4 & 1.48 \\
8 & 563.3 & 564.7 & 0.25 \\
9 & 578.6 & 608.4 & 4.90 \\
10 & 609.6 & 636.6 & 4.25 \\
\hline
\end{tabular}

It is known from Table 2 that the maximum relative error of gearbox model natural frequency respectively from experiment and numerically calculation is less than $5 \%$. And each order vibration mode is consistent with experimental results. It indicates the error of gearbox finite element model is acceptable.

\subsection{Description of housing deformation}

Flexible deformation of gearbox is described by modal basic function and modal coordinate in essence. By the conversion from modal coordinate to generalized coordinate, housing flexibility is integrated with system dynamics equation. Therefore, dynamics coupling of housing and transmission system is realized mathematically.

\section{Coupled Mathematic Model}

Gear mesh force and engine excitation are transmitted to gearbox housing by bearing system. And housing dynamic responses and dynamic characteristics of gear train system interact with the assist of bearing. Therefore, how to expound the mathematic relationship of housing, bearing and transmission shafts is crucial to construct dynamic coupled model.

Contacting characteristics of bearings are described as stiffness and dampness matrix. Flexible housing and transmission shafts consist of thousands of discrete mass elements. Elements are incorporated by kinematics constraint of each couple nodes. Therefore, generalized coordinate of each node is expressed by mode 
parameters; kinematics constraint is equivalent to stiffness and dampness element. Dynamics model of housing-bearing-shaft in one direction is shown in Fig.5.

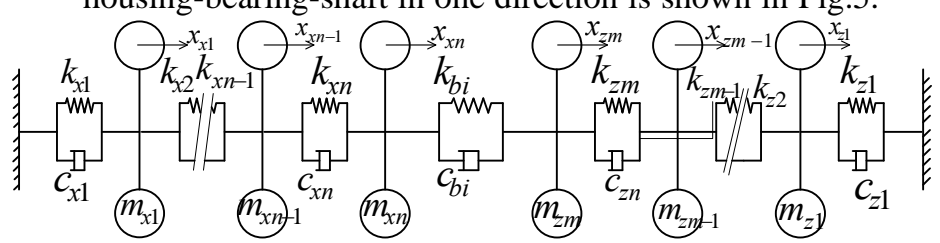

Fig.5. Dynamic model of housing-bearing-shaft The corresponding mathematic model is:

$$
\left\{\begin{array}{l}
m_{x 1} \ddot{x}_{x 1}=\left(k_{x 1}+k_{x 2}\right) x_{x 1}-k_{x 2} x_{x 2}+\left(c_{x 1}+c_{x 2}\right) \dot{x}_{x 1}-c_{x 2} \dot{x}_{x 2} \\
\cdots \\
m_{x n} \ddot{x}_{x n}=\left(k_{x n}+k_{b i}\right) x_{x n}-k_{x n} x_{x n-1}-k_{b i} x_{z n}+\left(c_{x n}+c_{b i}\right) \dot{x}_{x n}-c_{x n} \dot{x}_{x n-1}-c_{b i} \dot{x}_{z n} \\
m_{z m} \ddot{x}_{z m}=\left(k_{z m}+k_{b i}\right) x_{z m}-k_{b i} x_{x n}-k_{z m} x_{z m-1}+\left(c_{z m}+c_{b i}\right) \dot{x}_{z m}-c_{b i} \dot{x}_{x n}-c_{z m} \dot{x}_{z m-1} \\
\cdots \\
m_{z 1} \ddot{x}_{z 1}=\left(k_{z 1}+k_{z 2}\right) x_{z 1}-k_{z 2} x_{z 2}+\left(c_{z 1}+c_{z 2}\right) \dot{x}_{z 1}-c_{z 2} \dot{x}_{z 2}
\end{array}\right.
$$

where subscript $\mathrm{n}$ shows the number of housing elements, subscript $m$ shows the number of shaft elements, subscript $\mathrm{x}$ denotes the housing, subscript $\mathrm{b}$ denotes bearings and subscript $\mathrm{z}$ denotes shafts. This model is used to obtain the correlation characteristics of housing deformation and dynamic behaviors of gear train system.

\section{Model Construction}

\subsection{Transmission shaft}

To investigate the effect on system dynamic characteristics caused by transmission shafts flexibility, discrete disposal of shaft components is executed applying aforementioned finite element method. After the establishment of finite element analysis model and definition of boundary conditions, vibration mode is analyzed. Fig.6 shows the second constraint mode of the $2^{\text {nd }}$ shaft. Modal parameters is recognized in dynamics environment by coordinate conversion.

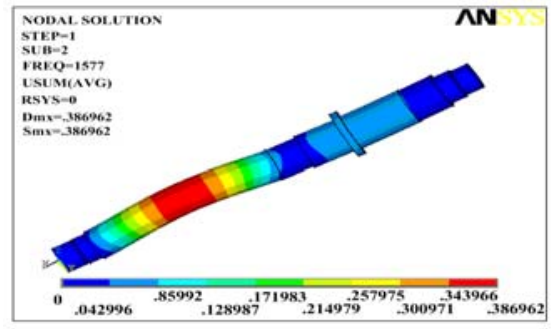

Fig.6. the $2^{\text {nd }}$ mode of the $2^{\text {nd }}$ shaft

\subsection{Engine}

Because of the periodic motivation torque generated by gas pressure and the inertia force and gravity of reciprocating components, engine torsional excitation is pulsatile under stable condition. On the hypothesis of ignoring the deformation, surface friction and wear, its output torque is:

$$
T=T_{0}+\sum T^{\prime}
$$

where $T_{0}$ is the average output torque of engine,

$\sum T^{\prime}$ is torsional vibration excitation of crankshaft system, mainly including periodic torque generated by combustion pressure.

Because each component in ADAMS(Automatic Dynamic Analysis of Mechanical System, a multi-body dynamics software) possesses its own accurate mass and inertia, the effect of variable inertia on engine dynamic characteristics is taken into consideration automatically. ADAMS is selected as the numerically simulation environment. According to AKISPL interpolation function, combustion pressure of each cylinder is calculated based on indicator diagram from test data as the ignition order. A certain type four-stroke and eightcylinder engine matches the research gearbox.

Crankshaft flexibility characteristics are mathematically described and dynamic model of sliding bearing (shown as Fig.7) is modified to be used in dynamics conveniently. The engine analysis model is constructed based on the dynamics description of the connection between each component.

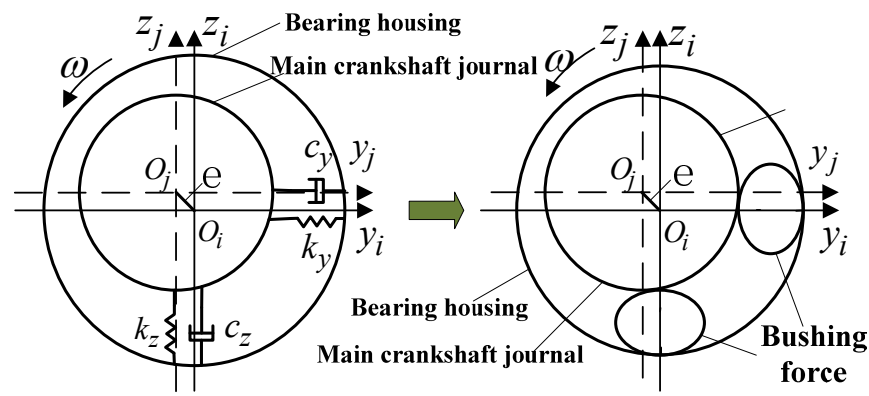

Fig.7. Schematic diagram of flexible support

The mathematic model is

$\left\{\begin{array}{l}m \ddot{y}_{j}(t)+c_{y} \dot{y}_{j}(t)+k_{y}\left[y_{j}(t)-y_{i}(t)\right]=m \omega^{2} e \cos \omega t \\ m \ddot{z}_{j}(t)+c_{z} \dot{z}_{j}(t)+k_{z}\left[z_{j}(t)-z_{i}(t)\right]=m \omega^{2} e \sin \omega t\end{array}\right.$ 
where $O_{i}$ is the center of bearing; $O_{j}$ is the center of main crankshaft journal; $m$ is the mass of crankshaft; $c_{y}$ and $c_{z}$ are the radial support damping coefficient; $k_{y}$ and $k_{z}$ are the radial support stiffness coefficients; $e$ is the eccentricity of shaft journal relative to the center of bearing; $\omega$ is the angular velocity of the crankshaft.

After setting engine working boundary conditions and ADAMS solver parameters, crankshaft output torque in one period is numerically acquired.

\subsection{Gear pair}

Gear joint in ADAMS only guarantees the kinematics relation of gear pair. And it can not reflect the real dynamic mesh state. So it is necessary to develop the dynamics model of gear set. A medium gear without mass is built. It guarantees kinetics relationship with driving gear applying correlative constraints.

Torsional spring connecting neutral gear and driven gear can portray dynamics behaviour caused by inner excitations. Fig.8 shows the modified model of gear set.

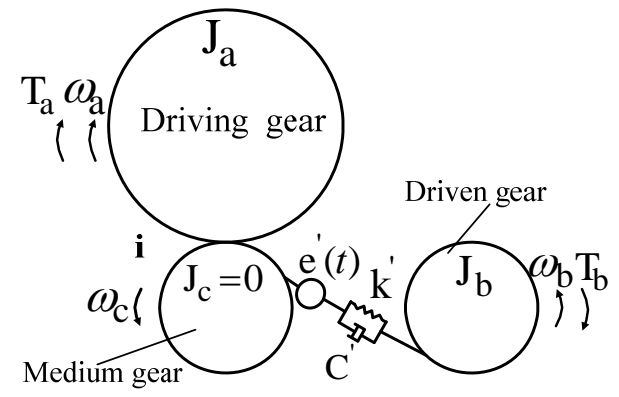

Fig.8. Modified dynamic model of gear set

Mathematic model is:

$$
\left\{\begin{array}{l}
T_{a}-\frac{\left[K^{\prime} e^{\prime}(t)+K^{\prime}\left(\theta_{c}-\theta_{b}\right)+C^{\prime}\left(\dot{\theta}_{c}-\dot{\theta}_{b}\right)\right]}{i}=J_{a} \ddot{\theta}_{a} \\
{\left[K^{\prime} e^{\prime}(t)+K^{\prime}\left(\theta_{b}-\theta_{c}\right)+C^{\prime}\left(\dot{\theta}_{b}-\dot{\theta}_{c}\right)\right]-T_{b}=J_{b} \ddot{\theta}_{b}}
\end{array}\right.
$$

where $T_{a}$ is the driving torque; $T_{b}$ is the driven torque; $\theta_{a}$ is the rotational angle of driving gear; $\theta_{b}$ is the rotational angle of neutral gear; $\theta_{c}$ is the rotational angle of driven gear; $K^{\prime}$ is the equivalent torsional stiffness coefficient, $K^{\prime}=K R_{b}^{2} ; C^{\prime}$ is the equivalent torsional damping coefficient, $C^{\prime}=C R_{b}^{2} ; e^{\prime}(t)$ is the equivalent mesh error, $e^{\prime}(t)=e(t) / R_{b} ; R_{b}$ is the basic circle radius of driven gear; $i$ is the gear ratio.

3-D dynamic contact finite element method can really reflect the mesh state of gear set. The deformation of mesh teeth can be numerically calculated in time. Aimed at the transmission gear sets in Fig.1, the analysis model of gear sets is constructed in software LS-Dyna (shown as in Fig.9).

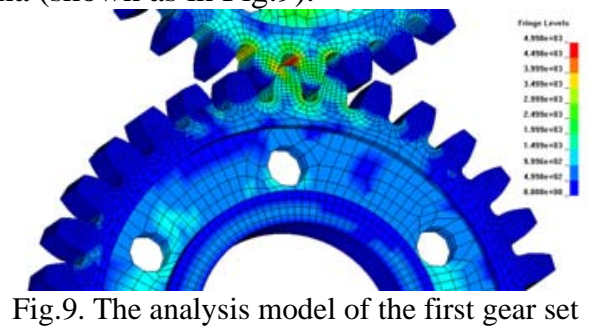

According to the gained combinatorial deformation of teeth, gear meshing integrated stiffness $\mathrm{K}$ is numerically calculated.

The error excitation is the displacement dynamic excitation in essence. And it's studied instead of mesh wrap. Mesh wrap is expressed by harmonic function.

From reference 15, meshing damping is:

$$
C=2 \xi_{g} \sqrt{\frac{K R_{a}^{2} R_{b}^{2} J_{a} J_{b}}{R_{a}^{2} J_{a}+R_{b}^{2} J_{b}}}
$$

where $\xi_{g}$ is the damping ratio. According to the calculation analysis of R.Kasuba and K.L.Wang, $\xi_{g}$ is generally from 0.03 to 0.17 .

\subsection{Supporting bearing}

Contact dynamic characteristic of rolling bearing is taken into account. In MSC.ADAMS environment, medium parts without mass are created where a bearing is. Revolution joint between shaft and medium part and planer joint between medium parts and bearing seat are created, which ensures bearing kinematics movement. Bearing dynamic characteristic is simulated by creating bushing between transmission shaft and spring element seat. As shown in Fig.10, dynamic model of shaftbearing system is constructed. 


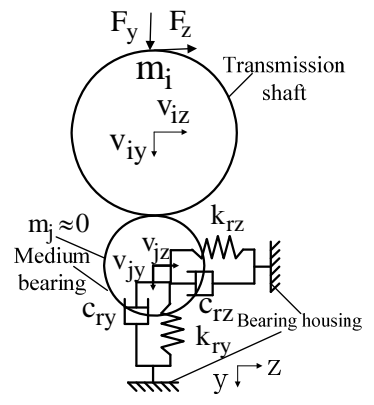

Fig.10. Dynamic model of bearing-shaft system The mathematic model is:

$\left\{\begin{array}{l}F_{y}=m_{i} \dot{v}_{i y}+c_{r y} v_{i y}+k_{r y} \int v_{i y} d t \\ F_{z}=m_{i} \dot{v}_{i z}+c_{r z} v_{i z}+k_{r z} \int v_{i z} d t\end{array} \quad\left\{\begin{array}{l}v_{i y}=v_{j y} \\ v_{i z}=v_{j z}\end{array}\right.\right.$

where $F_{y}$ and $F_{z}$ are the support forces in $\mathrm{y}$ and $\mathrm{z}$

direction respectively; $m_{i}$ is the mass of transmission

shafts; $v_{i y}$ and $v_{i z}$ are the relative velocity of transmission shaft versus bearing block in $\mathrm{y}$ and $\mathrm{z}$ direction respectively; $\mathrm{v}_{\mathrm{jy}}$ and $\mathrm{v}_{\mathrm{jz}}$ are the relative velocity of neutral part versus bearing block in $\mathrm{y}$ and $\mathrm{z}$ direction respectively; $c_{r y}$ and $c_{r z}$ are the damping coefficients in $\mathrm{y}$ and $\mathrm{z}$ direction respectively; $k_{r y}$ and $k_{r z}$ are the stiffness coefficients in $\mathrm{y}$ and $\mathrm{z}$ direction respectively.

Contact dynamic model of ball bearing in EHL condition is constructed applying EHL theory, dynamics of rolling bearing and Hertz contact theory. Timedependent curves of support stiffness coefficient and damping coefficient are numerically calculated using MATLAB based on characteristic parameters of lubrication and material, bearing structure parameters.

\subsection{System model}

Fig.11 shows the dynamics model of gearbox at a certain shift in consideration of the housing flexibility.

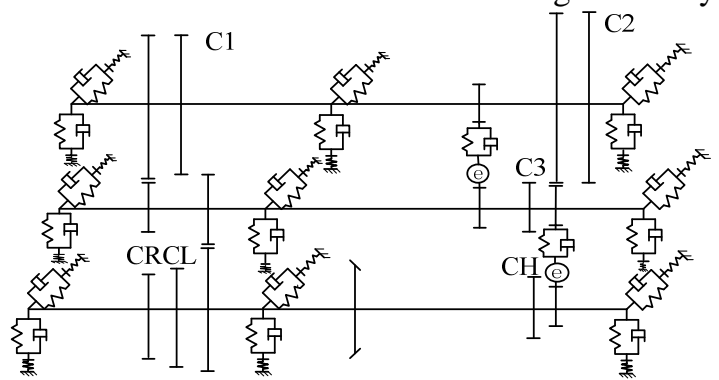

Fig.11. Dynamics model of gearbox
According to the boundary condition, system dynamics function is obtained based on each dynamic sub-structure in ADAMS. After solving the dynamics equation, dynamic responses of any node in gearbox housing and transmission shafts are gained. Fig.12 shows the dynamic analysis model of gearbox.

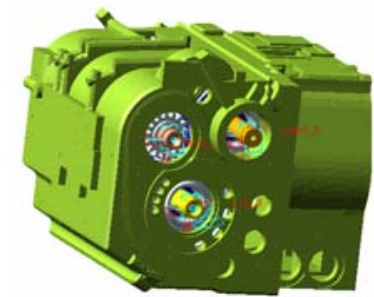

Fig.12. analysis model of gearbox

\section{Simulation Analysis}

To obtain the accurate dynamic response, it is crucial to bring multi-source excitations to the system analysis model. Engine fluctuate excitation is equivalently brought to the input port of system. Road load is defined at the output port to maintain dynamics balance. Gear inner excitations and support stiffness and damper coefficients are respectively put into corresponding mathematical model. Visual simulation of gearbox housing is realized. Coupled dynamic characteristics are deeply discussed under the some stable shift (shown as Fig.1).

\subsection{Effect of housing flexibility on dynamic characteristic of train system}

\subsubsection{Kinematics parameters}

The output speed is one of the most important kinematics parameters. It is essential to analyze the effect of housing flexibility.

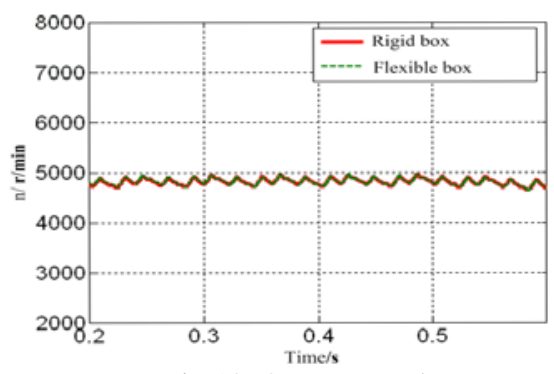

Fig.13. Output speed 
It is known from Fig.13 that two curves are consistent with each other and both maximum errors comparison with the theoretical value are $4.61 \%$, which are acceptable. It indicates that housing flexibility has no effect on system output speed. Dynamics parameters have relationship only with power condition of system and parameters characteristics of transmission components.

\subsubsection{Load characteristic}

Dynamic loads in gearbox are important parameters to reflect the system characteristics. Gear mesh force is one of main causes to generate vibration and chaos. Bearing support force is boundary condition for optimization design of gearbox and shafts. It is necessary to research the effect of housing flexibility.

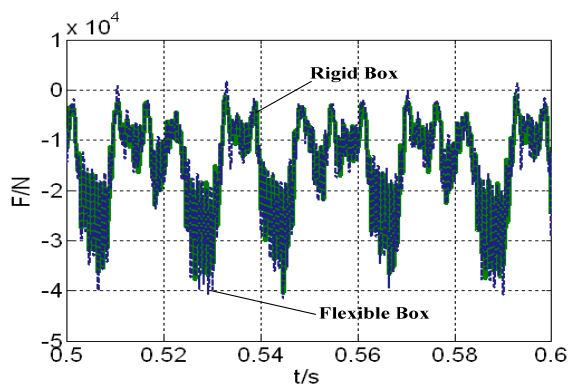

Fig.14. Gear mesh force

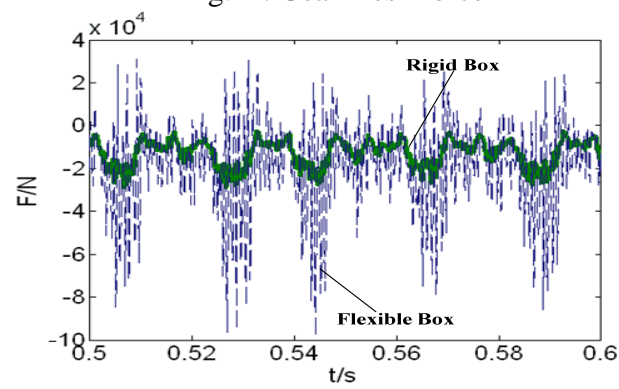

Fig.15. Bearing support force

From Fig.14, it is known that gear mesh force has little relationship with housing flexibility. Two curves have the same variable trend along time coordinate, which are accordance with the gear dynamic mesh condition. The average value is $-17757.90 \mathrm{~N}$ and the maximum value is $40461.36 \mathrm{~N}$ under the condition of rigid housing. When considering the housing flexibility, the average value is $-17907.41 \mathrm{~N}$ and the maximum value is $41629.5 \mathrm{~N}$. Their relative errors are respectively $0.84 \%$ and $2.89 \%$. It demonstrates that structural parameters of gear sets and power boundary conditions are the main factors to determine the dynamic mesh characteristics.

Fig. 15 shows that bearing support forces both fluctuate as the same period, although there is a great difference in amplitude. The support force varies drastically and the average value is $-18497.61 \mathrm{~N}$ considering housing flexibility. Under the situation of rigid housing, the support force changes evenly and the maximums are close to each other. The average value is $-12951.94 N$. It indicates that housing flexibility has a great effect on the contact dynamic characteristics of bearing. The existence of big difference results from the deformation of bearing interface nodes caused by the surface nodes deformation of housing according to the displacement constraint condition.

\subsubsection{Dynamic response}

Dynamic response is one of important dynamic characteristics of gearbox. The node of transmission shaft where is the center of gear b is selected to be studied.
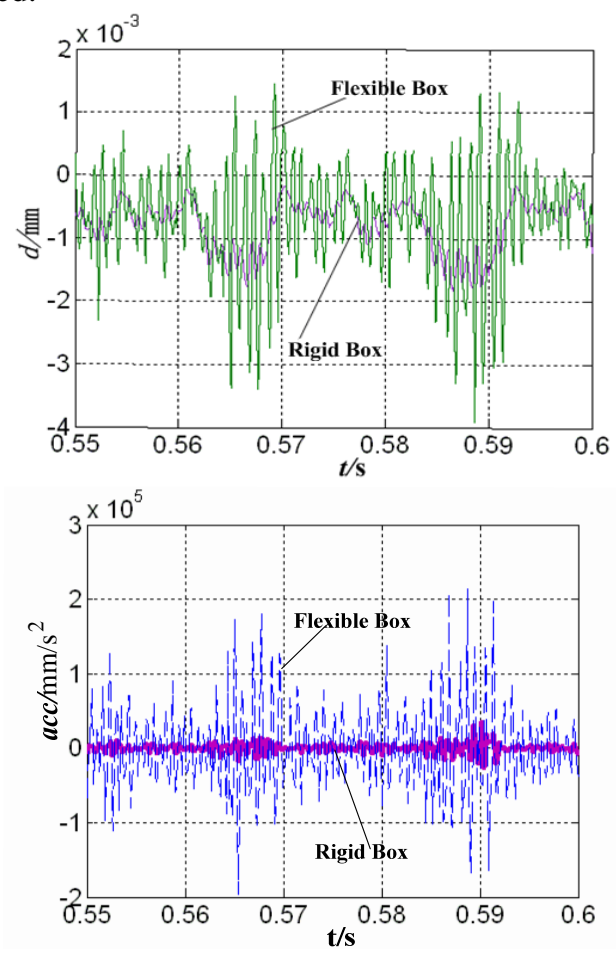

Fig.16. displacement and acceleration response of nodes in shaft

It is shown in Fig.16 that housing flexibility has a great impact on the dynamic response of transmission 
system. The fluctuating range of dynamic response (displacement and acceleration) significantly expands and varied sharply. It demonstrates that deformations of gearbox housing and dynamic responses of transmission system are interactional. Gearbox housing behaves dynamics characteristics under bearing support forces. It also has effect on the dynamic behaviors of transmission system. And the additional dynamic responses are generated.

\subsection{Dynamic response analysis of housing surface}

Applying the same boundary conditions, structural analysis is adopted to distinguish the weak region. The comprehensive strain nephogram of the gearbox housing (shown as Fig.18) is gained. And it is found that the region around node 23541 possesses the maximum strain. So this district of housing is selected to be studied. The Fig.17 shows the vibration displacement of node 23451 in both time and frequency domain.

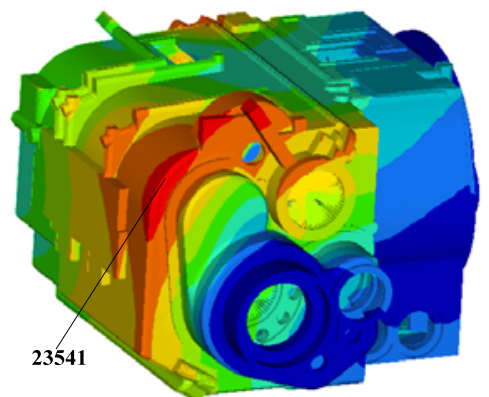

Fig.17. comprehensive strain nephogram of the gearbox housing
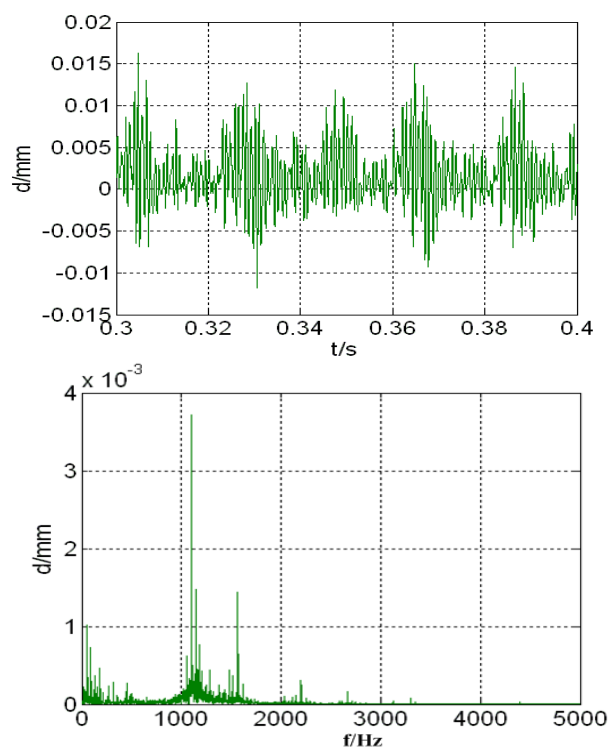

Fig.18. Vibration displacement of node 23451 in Z direction

It is shown in Fig.18 that vibration displacement fluctuates with time and the phenomenon of attenuation tendency doesn't exist in time domain because of multisauce excitations. And the maximum is $0.016 \mathrm{~mm}$. In the frequency domain, the maximums of displacement response occur at around $1100 \mathrm{~Hz}$ and $1564.4 \mathrm{~Hz}$ consistent with the mesh frequency of transmission gears teeth. In the low-frequency region(less than $500 \mathrm{~Hz}$ ), some peak values also appear and values are apparently lower. And these are caused by dynamic motivation of engine. It suggests that gear inner excitations have a greater effect on the dynamic response of gearbox housing than the pulsatile torque of engine.

\section{Test Validations}

To validate the accuracy of coupled analysis model and numerically simulation results, the test method is adopted. The test data is used for dynamic design and optimization of housing structure.

Nodes in the region having stronger vibration are selected as measured points according to the analysis of housing dynamic response. Fig. 19 shows the arrangement of measured points. 


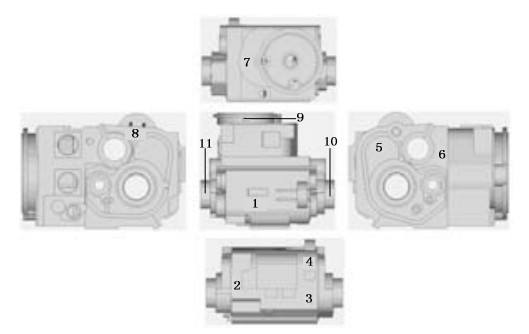

Fig.19. The arrangement of measured points

Test equipments are the same as what are used in the aforementioned modal test. To reflect the real vibration of gearbox housing, tracked vehicle equipped the power transmission system as shown in Fig.1 on road test is applied. And the concrete road is adopted. Acceleration responses are measured in different shift and input speed. Dynamic analysis model in the $4^{\text {th }}$ shift with the input speed of 2000r/min is discussed via the comparison of test data and CAE results.

Because of simulation model not including front and rear transmission, it is essential to establish the numerical filter. Concrete road is relatively favorable. Road resistance coefficient is set as 0.04 . After construction of the dynamic model in the $4^{\text {th }}$ shift with the input speed of 2000r/min, simulation results are validated by research on the dynamic characteristics of $6^{\text {th }}$ and $8^{\text {th }}$ measured points.

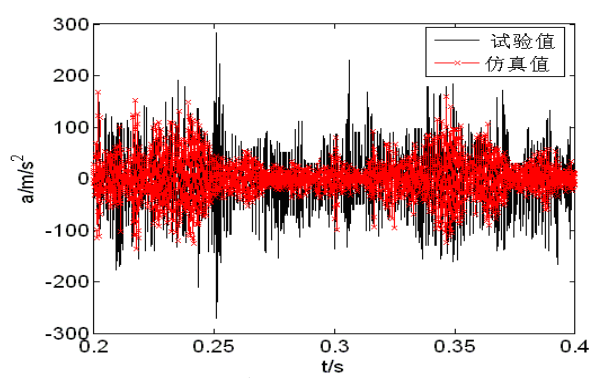

a) The $6^{\text {th }}$ measurement point

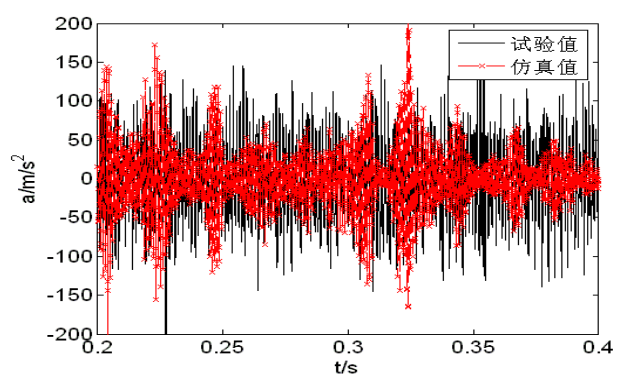

b) The $8^{\text {th }}$ measurement point Fig.20. Time domain

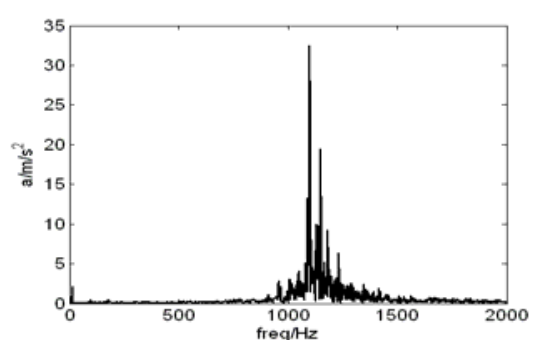

a) Simulation results of the $6^{\text {th }}$ measurement point

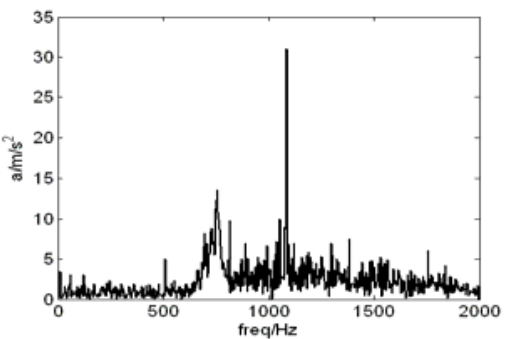

b) Test results of the $6^{\text {th }}$ measurement point

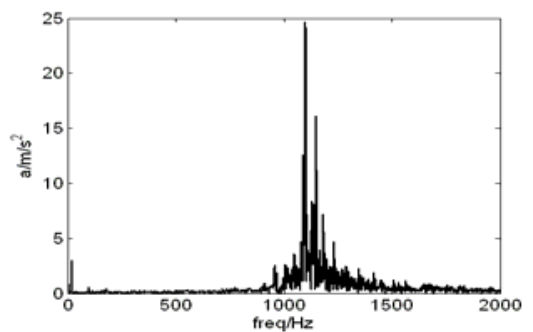

c) Simulation results of the $8^{\text {th }}$ measurement point

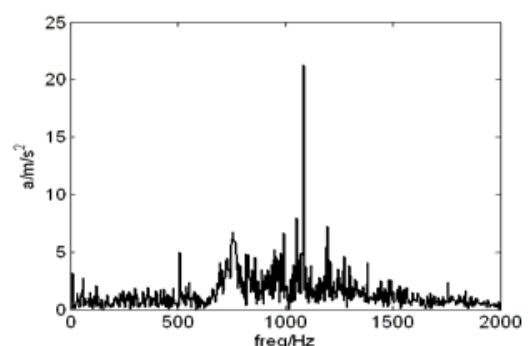

d) Simulation results of the $8^{\text {th }}$ measurement point Fig.21. Frequency domain

It is known from Fig.20 and Fig.21 that the gearbox housing acceleration response respectively from the numerically simulation and test under multisource excitations are consistent with each other. Simulation results fluctuate evenly and include few random factors. The root mean square values of normal vibration acceleration of each measurement point in simulation and test condition are shown in Table 3. 
Table 3. Root mean square values of normal vibration acceleration

\begin{tabular}{|c|c|c|c|c|c|c|c|}
\hline Measure & $2^{\text {nd }}$ & $3^{\text {rd }}$ & $4^{\text {th }}$ & $5^{\text {th }}$ & $6^{\text {th }}$ & $7^{\text {th }}$ & $8^{\text {th }}$ \\
\hline \multicolumn{8}{|l|}{ Simu } \\
\hline & 35.2 & 20.2 & 22.7 & 64.1 & 58.6 & 68.6 & 34.3 \\
\hline \multicolumn{8}{|l|}{-lation } \\
\hline Test & 46.1 & 32.5 & 37.4 & 98.6 & 89.4 & 95.1 & 45.9 \\
\hline Error & $23.7 \%$ & $37.9 \%$ & $39.3 \%$ & $34.9 \%$ & $34.5 \%$ & $27.7 \%$ & $25.3 \%$ \\
\hline
\end{tabular}

Root mean square values of normal vibration acceleration in test are higher than simulation results. The maximum error is $39.3 \%$. The main causes are analyzed as follow:

(1) Because of the effect of noise, lower peaks are distributed in the whole frequency domain in the test.

(2) Simulation model does not take the road roughness into consideration. Road roughness induces the normal vibration in the test.

(3) Friction and impact between each interior component and most stiffness and damp elements of the vehicle are neglected in simulation.

Via the comparison, the accuracy of model and simulation results is validated. It provides the important instructions for the structural design of gearbox housing.

\section{Conclusions}

This paper presented a new method to study on the coupled vibration characteristics of gearbox. Both numerical and test mode were deeply analyzed. Finite element model of gearbox housing was validated by the comparison of experimental data and numerical calculation results. It was the basis for the construction of gearbox model. Multi-source excitations were theoretically analyzed and numerically obtained to provide boundary conditions. Non-linear dynamics coupled model of gearbox in consideration of housing and transmission shafts flexibility was constructed. It broke through the localization that dynamic characteristics of gear transmission system and gearbox housing are separately analyzed. It proved the feasibility of analyzing the coupled dynamic characteristics of housing and train system. The coupled dynamic characteristics were deeply discussed. Vehicle test on road was adopted. The model and simulation results were validated. It indicated the analysis method was reasonable. It could also provide the important instructions for dynamic design of gearbox housing.

\section{Acknowledgements}

This work was partially supported by Natural Nature Science Foundation of China (51075033, 50905018). The authors would like to express gratitude to its financial support.

\section{References}

1.Wuhong Wang, Qi Cao, Katsushi Ikeuchi, Heiner Bubb, Reliability and safety analysis methodology for identification of drivers' erroneous actions, International Journal of Automotive Technology, 11(6) (2010),873-881.

2.Wuhong Wang, Yan Mao, Jin Jing, Xiao Wang, Hongwei Guo, Xuemei Ren, Ikeuchi Katsushi, Driver's various information process and multi-ruled decision-making mechanism: a fundamental of intelligent driving shaping model, International Journal of Computational Intelligence Systems, 4(3) (2011),297-305.

3.Y.Wang, W.J.Zhang: Stochastic vibration model of gear transmission systems considering speeddependent random errors. Nonlinear dynamics 17(1998):187-203.

4. Grzegorz Litak, Michael I.Friswell: Dynamics of a gear system with faults in meshing stiffness. Nonlinear dynamics 41(2005):415-421.

5.Tahar Fakhfakh, Hakher Chaari, Mohamed Haddar. Numerical and experimental analysis of a gear system with teeth defects. Int J Adv Manuf Technol 25(2005):542-550.

6.Chang-Jian, C.W., Chen, C.K.: Bifurcation and chaos of a flexible rotor supported by turbulent journal bearings with non-linear suspension. Trans. IMechE Part J.: J. Eng. Tribol.220(2006), 549-56.

7.Chang-Jian, C.W., Chen, C.K.: Bifurcation and chaos analysis of a flexible rotor supported by turbulent long journal bearings. Chaos Solitons Fractals 34(2007), 1160-1179.

8.Chang-Jian, C.W., Chen, C.K.: Nonlinear numerical analysis of a flexible rotor equipped with squeeze 
couple stress fluid film journal bearings. Acta Mech. Solida Sin. 20(4) (2007),283-290.

9.Saeed Ebrahimi, Peter Eberhard. Rigid-elastic modeling of meshing gear wheels in multibody systems. Multibody Syst Dyn, 16(2006):55-71.

10.Chan IL Park: Vibration from a shaft-bearing-plate system due to an axial excitation of helical gears. Journal of Mechanical Science and Technology, 20(12) (2006):2105-2114.

11.Mohamed Slim Abbes, Thar Fakhfakh, Mohamed Haddar, Aref Maalej: Effects of transmission error on the dynamic behavior of gearbox housing. Int $\mathrm{J}$ Adv Manuf Technol,34(2007):211-218.

12. LI Runfang, YANG Chengyun, LIN Tengjiao, et al. Finite element simulation of the dynamical behavior of o speed-increase gearbox. Journal of Materials Processing Technology, 150(2004):170-174.

13.LIU Wen, LIN Tengjiao, LI Runfang, et al. Finite element analysis of dynamic response and structure borne noise of gearbox Journal of Chongqing University-English Edition, 6(2) (2007):119-124.

14.YANG Yang. Research on the structure topology optimization of certain integrated transmission case. Master Thesis at Beijing Institute of Technology, Beijing,China,2007.

15.YANG Chengyun. Study on coupled vibration response and anti-impact property of gear transmissions system. PHD Thesis at Chongqing University,Chongqing,China,2006.

16.LI Shaobin. Study on coupled thermo-elastic deformation and nonlinear dynamic emulate about high-speed, heavy-load gear transmissions system. PHD Thesis at Chongqing University, Chongqing, China,2004.

17.LI Runfang, WANG Jian-jun. Dynamics of Gear System . Science Press, Beijing,China,1997. 